\title{
Review Article \\ Circulating Resistin Levels and Risk of Colorectal Cancer: A Meta-Analysis
}

\author{
Gui Yang, ${ }^{1}$ Wei Fan, ${ }^{2}$ Baohong Luo, ${ }^{2}$ Zhigao Xu, ${ }^{3}$ Ping Wang, ${ }^{2}$ Shihui Tang, \\ Peipei $\mathrm{Xu}^{2}{ }^{2}$ and Mingxia $\mathrm{Yu}^{1,2}$ \\ ${ }^{1}$ Department of Clinical Laboratory, Zhongnan Hospital of Wuhan University, Wuhan 430071, China \\ ${ }^{2}$ Department of Center for Gene Diagnosis, Zhongnan Hospital of Wuhan University, Wuhan 430071, China \\ ${ }^{3}$ Department of Pathology, Zhongnan Hospital of Wuhan University, Wuhan 430071, China \\ Correspondence should be addressed to Mingxia Yu; dewrosy520@163.com
}

Received 10 May 2016; Accepted 20 July 2016

Academic Editor: Oreste Gualillo

Copyright (C) 2016 Gui Yang et al. This is an open access article distributed under the Creative Commons Attribution License, which permits unrestricted use, distribution, and reproduction in any medium, provided the original work is properly cited.

\begin{abstract}
Objectives. Published data on resistin levels in patients with colorectal cancer (CRC) were conflicting and heterogeneous. We conducted a meta-analysis of observational studies to examine the association of circulating resistin levels with carcinogenesis of the CRC. Methods. Potentially eligible studies published up to November 2015 were searched through MEDLINE, EMBASE, Science Citation Index Expanded database, CNKI, and WanFang database. The pooled weighted mean differences (WMDs) with 95\% confidence intervals (CIs) calculated by fixed- or random-effect model were used to estimate the effects. Results. A total of 11 studies involving 965 patients were admitted in our meta-analysis. The pooled effects indicated that resistin levels were higher in CRC patients compared to healthy controls (WMD: $1.47 \mathrm{ng} / \mathrm{mL} ; 95 \%$ CI: 0.78 to 2.16), with significant heterogeneity across the studies $\left(I^{2}=72 \%, p<0.0001\right)$. Subgroup analyses and sensitivity analyses revealed that study quality, design, sample type, and resistin assays may account for this heterogeneity. No publication bias was observed. Conclusions. Our meta-analysis suggests that increased circulating resistin levels are associated with greater risk of colorectal cancer. Given the limited number of available studies and significant heterogeneity, larger well-designed randomized studies are warranted.
\end{abstract}

\section{Introduction}

Colorectal cancer is the third most common cancer and the third most common cause of high mortality cancer rates in the united states [1]. Although the incidence of CRC is $18 \%$ higher in developed regions in comparison with lowerincome areas [2], there is a rapid increase in developing countries in the past few decades, which makes it a major public health concern worldwide. CRC is recognized as a complex multipathway disease circled with chronic inflammation, metabolic syndrome, insulin resistance (IR), and obesity. The results from epidemiological evidence indicate that obesity is associated with increased incidence of CRC $[3,4]$. Recent studies suggest that adipocyte-derived secretory factors including adiponectin, leptin, resistin, visfatin, and numerous cytokines including TNF-alpha, IL-6, and IL-10 may be the key to link obesity with CRC, although underlying mechanisms remain unexplained [5-8].
Resistin, produced by adipose tissue whereas it is mostly secreted from monocytes and macrophages and involved in autocrine and paracrine cell signaling [9], has raised considerable research interest in recent years [10]. It is a cysteine rich 108 -amino-acid peptide with a molecular weight of $12.5 \mathrm{kDa}$, mapping to the p13.3 band of human chromosome 19 [11, 12] and circulating in low and high molecular weight forms [13]. As a member of resistin-like molecules (RELMs), which is a small family of secreted proteins associated with the activation of inflammatory [14], resistin can regulate inflammatory response, mediate metabolic disturbances and IR, promote cell proliferation, and enhance cancer progression [15], providing novel insights to obesity and colorectal cancer.

Although there are several systematic reviews evaluating the relationship between several adipocytokines and risk of CRC or CRA, including leptin and adiponectin [16-20], to the best of our knowledge, there is no meta-analysis on the associations of circulating resistin and CRC. In the past few years, 
some studies indicated a positive association between resistin levels and CRC [18, 21-28]; others failed to demonstrate such a relationship [29-32]. Seeing that controversial discrepancy, we summarized the evidence obtained from available data to assess resistin levels in patients with CRC.

\section{Materials and Methods}

Since we were evaluating observational studies, we conducted this meta-analysis in accordance with the Observational Studies in Epidemiology [33].

2.1. Search Strategy. A systematic literatures search from online databases including MEDLINE, EMBASE, and ISI Web of Knowledge was performed. The respective scientific studies up to November 20, 2015, were identified without language, year, or publication status restriction. Besides, we searched National Knowledge Infrastructure (CNKI) and WanFang database for Chinese literatures. The following terms were used as strategy: resistin OR adipokine OR adipocytokine OR adipocyte secreted factor (ADSF) OR FIZZ-3 OR obese protein, colorectal OR colon OR rectum, cancer OR tumor OR carcinoma OR neoplasm. Reference lists of identified literature were also checked for additional articles.

2.2. Inclusion and Exclusion Criteria. Eligible cohort and case-control studies reporting the association between serum or plasma resistin levels and colorectal cancer were included in this meta-analysis. We excluded studies as follows: (1) reviews or nonoriginal studies; (2) studies on animal or cell or tissue or genetic variation; (3) studies without controls or original data; (4) studies related to mechanism or prognosis or survival.

2.3. Data Extraction and Quality Assessment. The following information and data were extracted from each included study: (1) basic information such as first author's name, year of publication, and geographic location; (2) details of each trial, for example, study design and size, type of blood sampling, resistin detection method, and reagent; (3) characteristics of patients and controls including age, gender, BMI estimation, means, and standard deviation (SD) of resistin concentration. Two reviewers independently finished the extraction work and the disagreements were resolved by consensus or the third reviewer. Meanwhile, we systematically assessed the quality of each study with the Newcastle-Ottawa Scale (NOS) [34].

2.4. Statistical Analysis. All statistical analyses were conducted with Stata 11.0 (Stata, College Station, Texas, USA) and Review Manager 5.3 software (Cochrane Collaboration). Weighted mean differences (WMDs) and corresponding 95\% confidence intervals (CIs) were calculated based on the sample size, mean, and SD extracted from eligible studies. For five studies that only provided the median and range or interquartile range of outcomes, mean and SD were estimated, as described by Hozo et al. [35] and Liu et al.
[36]. The Cochran Q statistic and inconsistency index $\left(I^{2}\right)$ were used for examining the heterogeneity among studies, with the statistical significance level 0.05. Considering the presence of significant heterogeneity, we applied a random effects model for pooling effect, otherwise, a fixed-effects model.

In order to explore the sources of heterogeneity, we performed subgroup analysis by geographical area, study design, study size, quality, blood sample, resistin assay method, mean age, and mean BMI. Besides, several sensitivity analyses were conducted by omitting one study at a time, excluding estimated effect sizes and studies not adjusted for BMI. Publication bias was evaluated by funnel plot, complementary with Egger's regression method and Begg's rank correlation method.

\section{Results}

3.1. Search Results and Studies' Characteristics. A total of 122 potential records were detected by the primary database search and 78 were left after being autodeduplicated. By reading titles and abstracts for relevance, 54 reports were excluded because they were either review articles, mechanism studies, animal studies, or irrelevant to circulating resistin. Of the 24 records selected for detailed full-text evaluation, only 10 studies fulfilled our inclusion criterion. Three studies did not measure circulating resistin levels in healthy controls. Two trials were excluded because of one provided boxplot without data to describe distribution of serum resistin concentration and the other reported only odd ratio (OR) and $95 \%$ CI of the risk of colorectal cancer; we could not get primary data although we tried to contact the authors. Besides, a Chinese study that met the inclusion criterion was added. A flow diagram of selection strategy was presented in Figure 1.

Consequently, 11 studies including 965 patients with colorectal cancer and 1325 healthy controls were identified in this meta-analysis. Among the 11 studies, one was case-cohort study conducted in USA [31], while the other 10 studies were of case-control design conducted either in Asia [18, 21, 23, 25, $26,28]$ or in Europe $[24,27,29,32]$. Ten of the studies were matched for age and/or gender [18, 21, 23, 24, 26-29, 31, 32] and 6 of them were adjusted for BMI additionally [21, 23, 24, $26,27,31]$. The characteristics of each study were summarized in Table 1 . The quality assessments of 11 case-control studies were presented in Table 2 . The only case-cohort study [31] was finally awarded 7 stars based on NewcastleOttawa Scale (NOS) and specific assessment items were not listed.

3.2. Data Analysis. Three studies showed that patients with colorectal cancer had no significant difference in circulating resistin concentration compared to healthy controls $[29,31$, 32], while significant increase was presented in other nine studies $[18,21-28]$. The summary WMD of resistin levels between CRC patients and control group was $1.47 \mathrm{ng} / \mathrm{mL}$ (95\% CI: 0.78 to 2.16 ), and $z$-score for overall effect was $4.19(p<0.0001)$, which revealed a possibility that higher 


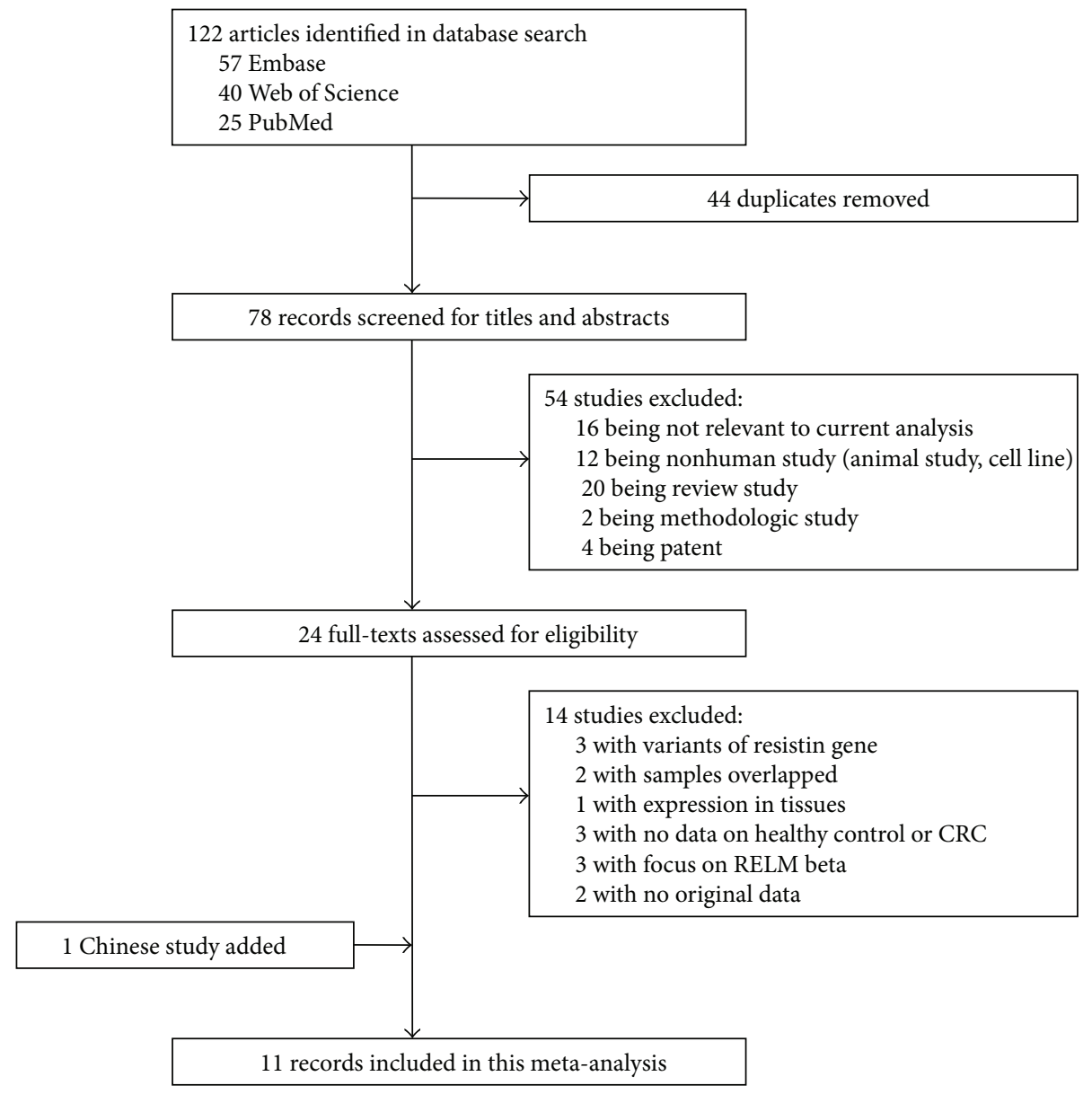

FIGURE 1: A flow diagram of selection strategy.

circulating resistin level was associated with higher risk of colorectal tumor. Significant heterogeneity was observed among studies and needed further analysis $\left(I^{2}=72 \%, p<\right.$ 0.0001; Figure 2).

3.3. Subgroups/Sensitivity Analysis. We subsequently conducted subgroups and sensitivity analysis to trace the potential sources of heterogeneity. In subgroup analysis, the summary WMD of circulating resistin levels between CRC patients and healthy controls did not differ substantially by mean age, sample size, and mean BMI. Thus, heterogeneity could not be explained by those variables. However, positive association was observed between resistin levels and risk of colorectal cancer in serum samples, studies with ELISA method, low quality studies, studies of Asian origin, and casecontrol design, indicating the possible origin of heterogeneity. Pooled WMD of resistin levels in serum samples (WMD, 1.97; 95\% CI, 1.18-2.75), studies with ELISA method (WMD, $1.68 ; 95 \%$ CI, $0.95-2.41$ ), low quality studies (WMD, 1.69; 95\% CI, 0.98-2.41), studies of Asian origin (WMD, 1.70; 95\% CI, $1.03-2.37$ ), and case-control design (WMD, 1.84 ; 95\% CI, 1.512.18) were stronger than in plasma samples (WMD, 0.59; 95\% CI, -0.39-1.57), studies with multiplex immunoassay (WMD,
$0.45 ; 95 \% \mathrm{CI},-0.50-1.40)$ and high quality studies (WMD, $1.22 ; 95 \% \mathrm{CI},-0.33-2.78$ ), studies of Western origin (WMD, 0.93 ; 95\% CI, $-1.20-3.05$ ), and case-cohort design (WMD, 0.50 ; $95 \%$ CI, $-0.52-1.52$ ). Results of subgroups analysis are presented in Table 3.

Three of 11 studies mentioned the relationship between circulating resistin levels and TNM stages of colorectal cancer. The results of those studies were inconsistent (Table 4). Pooled mean differences suggested that circulating resistin levels might not be significantly different in CRC stage II-IV patients (Figure 3).

In sensitivity analysis, there was no significant difference after deleting a single study at a time and calculating pooled WMD of the remainders (Figure 4). Neither separate analysis of studies matching for BMI yielded significant associations of resistin levels and colorectal cancer risk. While removing studies for which the mean and SD were estimated [23, 27, 29, 31, 32], a stronger association between resistin and CRC was noted (WMD, 2.10; 95\% CI, 1.25-2.95).

3.4. Publication Bias. We did not find distinct funnel plot asymmetry, raising a low probability of publication bias (Figure 5). Egger's regression test $(p=0.34)$ and Begg's 


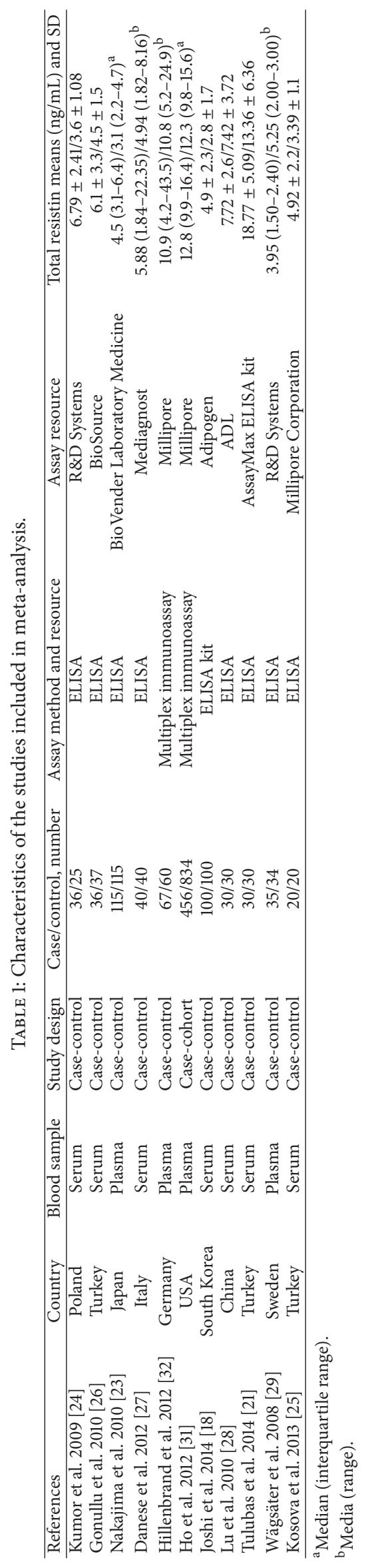




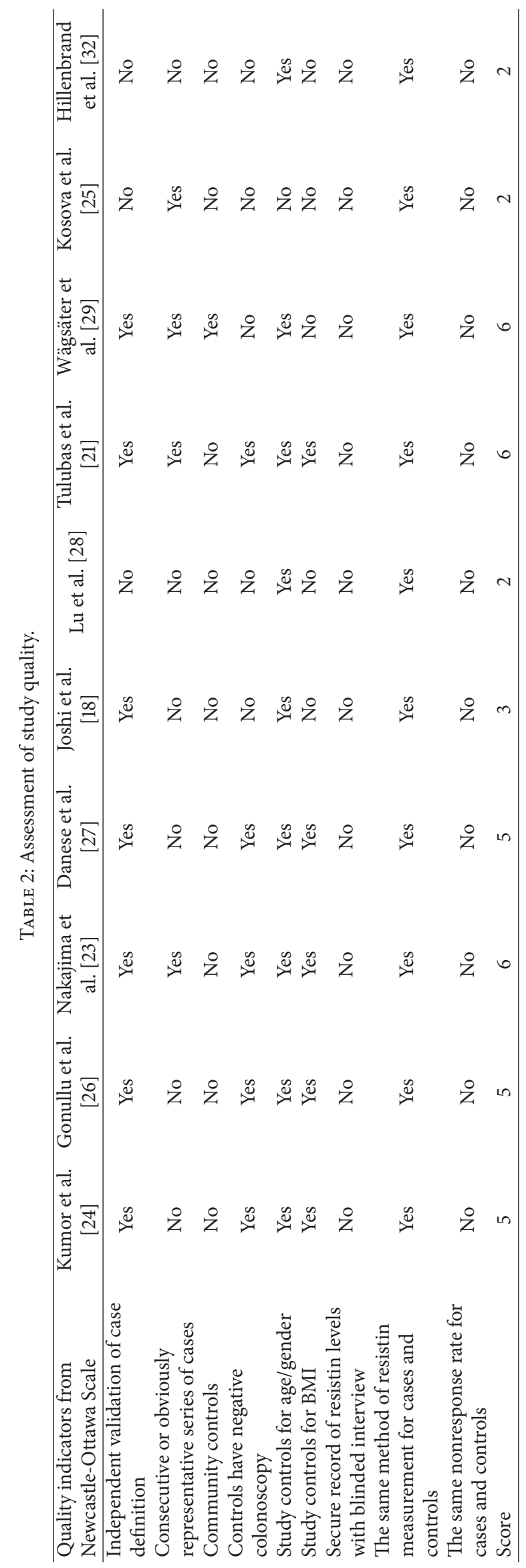






FIGURE 2: Forest plot of weighted mean differences (WMD) with 95\% confidence intervals (CIs) of circulating resistin levels between colorectal cancer patients and healthy controls.

adjusted rank correlation test $(p=0.78)$ verified that possibility (Figure 6).

\section{Discussion}

The results of this meta-analysis suggested that resistin levels were notably higher in patients with CRC than those in healthy controls, indicating that resistin levels may be positively correlated with risk of colorectal cancer, although there was significant heterogeneity among the studies. The effects of estimates were consistent for studies regardless of their sample size, mean age, mean BMI, and whether being adjusted for BMI or not. No publication bias was observed in our meta-analysis. Keeping in mind the role of resistin in linking obesity to colorectal cancer risk, as well as the inconsistent published results regarding the effect of resistin in this setting, this meta-analysis is of special importance.

Although the role of resistin in colorectal cancer is far from being elucidated, several mechanisms may be involved in explaining these outcomes. In vitro studies demonstrate that resistin has proinflammatory effects mediated by TLR4 receptor stimulation and $\mathrm{NF}-\kappa \mathrm{B}$ pathway [37, 38]. Other studies show that resistin regulates production of the matrix metalloproteinases (MMPs) and modulates the secretion of vascular endothelial growth factor (VEGF), which is considered of importance for promoting tumor invasion [39, 40]. An animal study, where the mice were dealt with to lack adipocyte-derived mouse resistin but be able to produce human resistin resulting in rapidly accelerated white adipose tissue (WAT) inflammation after the high-fat diet, indicated that resistin contributes to WAT inflammation and insulin resistance [41]. Furthermore, RETN (rs1862513) or -420C>G resistin gene polymorphism is associated with decreased CRC susceptibility [29, 42].

Recently, studies demonstrate that obesity is strongly related to CRC, which is involved in several mechanisms, such as obesity-related insulin resistance, oxidative stress [43], adipocytokine production [44], and insulin-like growth factor-1 (IGF-1) [45]. They are all responsible for cancer promoting effects, increasing tumor cell migration, and finally leading to tumor metastasis. The overexpression of resistin results in the accumulation of intracellular lipid and then leads to obesity-mediated insulin resistance and inflammation [46, 47]. Therefore, it is widely accepted that high resistin levels are related to inflammatory conditions and malignancies $[48,49]$.

However, we cannot ignore the significant heterogeneity across studies in the interpretation of the findings. To investigate potential sources, we performed subgroup and sensitivity analyses. Our study suggested that BMI may not be important in explaining the heterogeneity, since effect estimates showed no difference in different mean BMI subgroup and studies whether adjusting for BMI or not. However, the stronger and more detailed estimating factor for obesity including waist circumference and waist-to-hip ratio was not available in most involved studies, so effect of 
TABLE 3: Subgroup analysis of circulating resistin levels and colorectal cancer.

\begin{tabular}{|c|c|c|c|c|c|c|}
\hline \multirow{2}{*}{ Stratification group } & \multirow{2}{*}{ Data points $(N)$} & \multicolumn{2}{|c|}{ Cases } & \multirow{2}{*}{$\begin{array}{l}\text { Random effects } \\
\text { WMD (95\% CI) }\end{array}$} & \multicolumn{2}{|c|}{ Heterogeneity } \\
\hline & & CRCs & Controls & & $I^{2}(\%)$ & $p$ value \\
\hline All studies & 11 & 965 & 1325 & $1.47[0.78,2.16]$ & 72 & $<0.0001$ \\
\hline \multicolumn{7}{|l|}{ Study location } \\
\hline Western & 4 & 178 & 159 & $0.93[-1.20,3.05]$ & 83 & 0.0005 \\
\hline Asian & 6 & 331 & 332 & $1.70[1.03,2.37]$ & 57 & 0.04 \\
\hline USA & 1 & 456 & 834 & $0.50[-0.52,1.52]$ & - & - \\
\hline \multicolumn{7}{|l|}{ Study design } \\
\hline Case-control & 10 & 509 & 491 & $1.84[1.51,2.18]$ & 70 & 0.0004 \\
\hline Case-cohort & 1 & 456 & 834 & $0.50[-0.52,1.52]$ & - & - \\
\hline \multicolumn{7}{|l|}{ Sample size } \\
\hline$<100$ CRC cases & 8 & 294 & 276 & $1.48[0.38,2.57]$ & 75 & 0.0002 \\
\hline$\geq 100$ CRC cases & 3 & 671 & 1049 & $1.42[0.55,2.28]$ & 74 & 0.02 \\
\hline \multicolumn{7}{|l|}{ Blood sample } \\
\hline Plasma & 4 & 673 & 1043 & $0.59[-0.39,1.57]$ & 50 & 0.11 \\
\hline Serum & 7 & 292 & 282 & $1.97[1.18,2.75]$ & 68 & 0.004 \\
\hline \multicolumn{7}{|l|}{ Assay method } \\
\hline ELISA & 9 & 442 & 431 & $1.68[0.95,2.41]$ & 72 & 0.0004 \\
\hline Multiplex immunoassay & 2 & 523 & 894 & $0.45[-0.50,1.40]$ & 0 & 0.78 \\
\hline \multicolumn{7}{|l|}{ Study quality } \\
\hline Low (NOS score $\leq 5)$ & 7 & 329 & 312 & $1.69[0.98,2.41]$ & 62 & 0.01 \\
\hline High (NOS score $>5$ ) & 4 & 636 & 1013 & $1.22[-0.33,2.78]$ & 79 & 0.002 \\
\hline \multicolumn{7}{|l|}{ Mean age, years } \\
\hline$<60$ & 2 & 66 & 67 & $1.15[0.19,2.11]$ & 38 & 0.2 \\
\hline$\geq 60$ & 9 & 879 & 1238 & $1.56[0.65,2.47]$ & 79 & $<0.0001$ \\
\hline Not mentioned & 1 & 20 & 20 & $1.53[0.45,2.61]$ & - & - \\
\hline \multicolumn{7}{|l|}{ Mean BMI, kg/m² } \\
\hline$<25$ & 1 & 115 & 115 & $1.40[0.64,2.16]$ & 73 & 0.01 \\
\hline$\geq 25$ & 6 & 665 & 1026 & $1.83[0.54,3.12]$ & 79 & 0.0002 \\
\hline Not mentioned & 4 & 185 & 184 & $1.03[-0.14,2.21]$ & 73 & 0.01 \\
\hline
\end{tabular}

TABLE 4: Circulating resistin levels in TNM patients with colorectal cancer.

\begin{tabular}{|c|c|c|c|c|c|c|c|c|}
\hline Studies & Sample size & Stage 0 & Stage I & Stage II & Stage III & Stage IV & $p$ value & Detection methods \\
\hline Nakajima et al. 2010 [23] & 115 & $1.3 \pm 0.5$ & $1.6 \pm 0.5$ & $1.5 \pm 0.5$ & $1.5 \pm 0.6$ & $1.7 \pm 0.5$ & $<0.01$ & ELISA \\
\hline Gonullu et al. 2010 [26] & 33 & - & - & $6.01 \pm 3.18$ & $7.37 \pm 3.58$ & $5.79 \pm 3.96$ & - & ELISA \\
\hline Kumor et al. 2009 [24] & 36 & - & \multicolumn{2}{|c|}{$5.86 \pm 3.1$} & \multicolumn{2}{|c|}{$6.79 \pm 2.41$} & $>0.05$ & ELISA \\
\hline
\end{tabular}

visceral fat accumulation could not be checked. Furthermore, cachexia patients with advanced CRC have lower BMI, which may be a confounding factor. Notably, the low quality and case-control studies pooled stronger relationship between resistin levels and CRC. The only case-cohort study with high quality demonstrated that plasma level of resistin was not associated with CRC. The discrepancy highlights the need for well-designed prospective studies on resistin and colorectal cancer. Besides, sample type and detection method may also contribute to heterogeneity. It may be explained by the reason that circulating resistin levels are affected by different storage condition and sensibility of assays referring to the very low resistin concentration in human blood.

There are other limits which should be acknowledged in our analysis. First, because this meta-analysis is based on observational studies that have inherent bias, the causal relationship of higher resistin levels and higher risk of CRC may not exist. Second, we eventually included 11 studies and 965 CRC patients in our analysis encompassing a case-cohort study which consisted of almost half research patients; the numbers of studies classified into some subgroups were too small to perform a pooled analysis. Possibly because resistin 


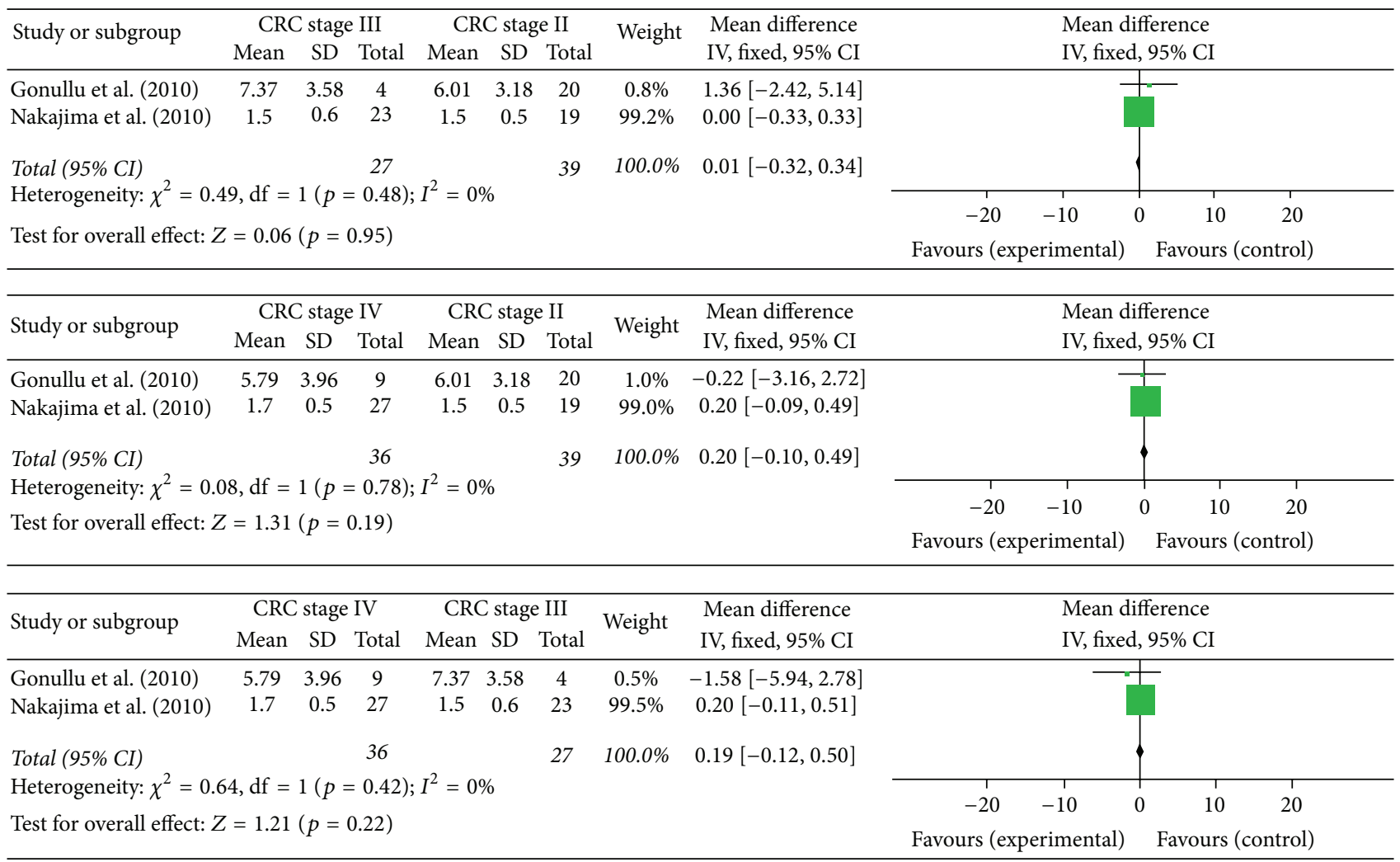

FIGURE 3: Forest plot of mean differences with its 95\% CIs of circulating resistin levels in CRC stage II-IV patients.

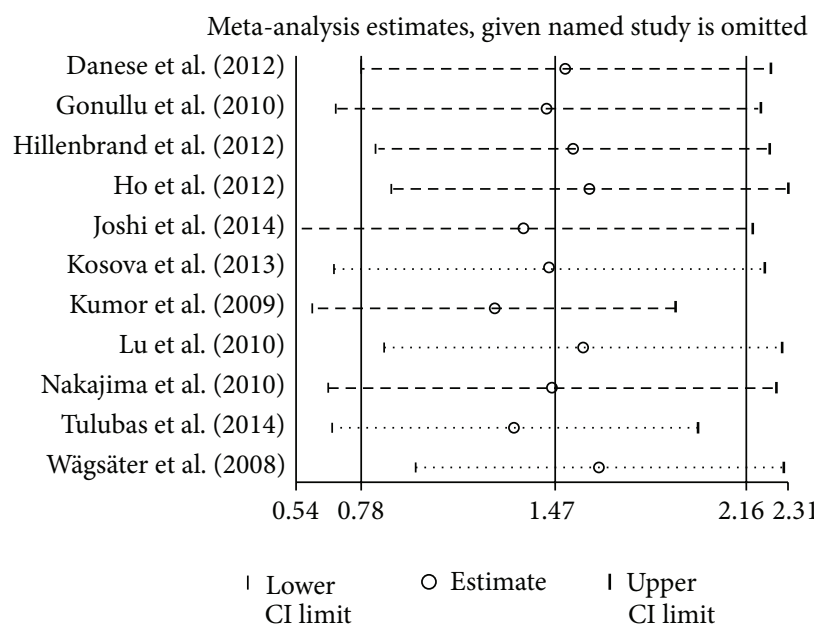

FIGURE 4: Influence analysis in studies of circulating resistin and colorectal cancer.

is newly discovered, researches with large sample size are lacked. Third, the estimates of mean and SD from median and range are a cause of bias as described in sensitivity analysis results. At last, we did not rule out residual confounders that may alter resistin levels, including lifestyle parameters such as smoking, alcohol, exercise, nutritional habits, and clinicopathological features such as tumor stage, localization, and type.
In conclusion, our study suggests that circulating resistin levels in patients with CRC are significantly higher than those in healthy controls, increasing the current understanding of the association between resistin levels and risk of colorectal cancer. Given the limitations mentioned above, larger well-designed randomized studies and even experimental researches are needed to confirm the effect of resistin levels in the development of colorectal cancer. 


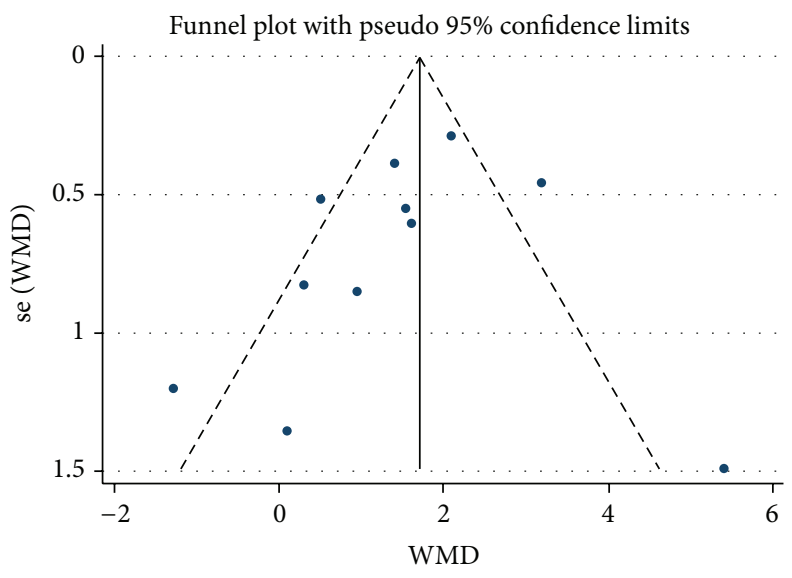

FIGURE 5: Funnel plot of publication bias.

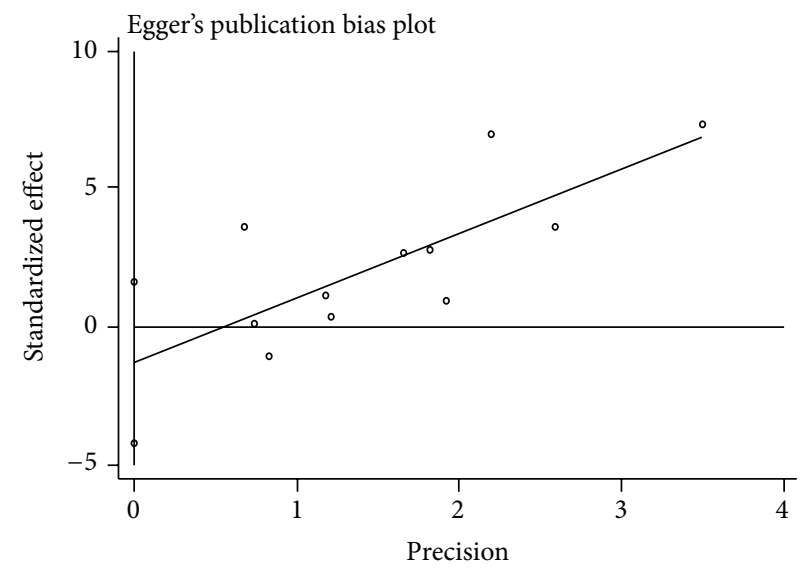

(a)

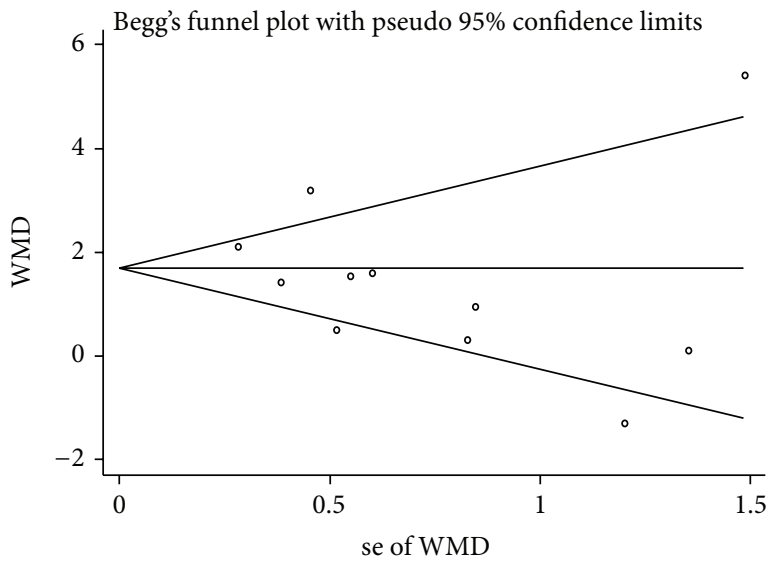

(b)

Figure 6: Egger's publication bias plot (a) and Begg's funnel plot (b).

\section{Competing Interests}

The authors declare no competing interests.

\section{Acknowledgments}

This study was supported by National Natural Science Funds (no. 81472033 and no. 30901308), the National Science Foundation of Hubei Province (no. 2013CFB233 and no. 2013CFB235), the Scientific and Technological Project of Wuhan City (no. 2014060101010045), and Hubei Province Health and Family Planning Scientific Research Project (WJ2015Q021).

\section{References}

[1] R. Siegel, C. Desantis, and A. Jemal, "Colorectal cancer statistics, 2014," CA: A Cancer Journal for Clinicians, vol. 64, no. 2, pp. 104117, 2014.

[2] R. Baena and P. Salinas, "Diet and colorectal cancer," Maturitas, vol. 80, no. 3, pp. 258-264, 2015.
[3] Y. Ma, Y. Yang, F. Wang et al., "Obesity and risk of colorectal cancer: A Systematic Review of Prospective Studies," PLoS ONE, vol. 8, no. 1, article e53916, 2013.

[4] S.-Y. Na and S.-J. Myung, "Obesity and colorectal cancer," Taehan Sohwagi Hakhoe Chi, vol. 59, no. 1, pp. 16-26, 2012.

[5] G. De Pergola and F. Silvestris, "Obesity as a major risk factor for cancer," Journal of Obesity, vol. 2013, Article ID 291546, 11 pages, 2013.

[6] C. R. Guffey, D. Fan, U. P. Singh, and E. A. Murphy, "Linking obesity to colorectal cancer: recent insights into plausible biological mechanisms," Current Opinion in Clinical Nutrition and Metabolic Care, vol. 16, no. 5, pp. 595-600, 2013.

[7] N. Vazzana, S. Riondino, V. Toto et al., "Obesity-driven inflammation and colorectal cancer," Current Medicinal Chemistry, vol. 19, no. 34, pp. 5837-5853, 2012.

[8] E. Yehuda-Shnaidman and B. Schwartz, "Mechanisms linking obesity, inflammation and altered metabolism to colon carcinogenesis," Obesity Reviews, vol. 13, no. 12, pp. 1083-1095, 2012.

[9] S. Jiang, D. W. Park, J.-M. Tadie et al., "Human resistin promotes neutrophil proinflammatory activation and neutrophil extracellular trap formation and increases severity of acute lung injury," Journal of Immunology, vol. 192, no. 10, pp. 4795-4803, 2014. 
[10] D. S. Popovic, D. Tomic-Naglic, and E. Stokic, "Relation of resistin, leptin and adiponectin-trinity of adipose tissue dysfunction assessment," European Journal of Internal Medicine, vol. 25, no. 6, pp. e80-e81, 2014.

[11] C. M. Steppan, E. J. Brown, C. M. Wright et al., "A family of tissue-specific resistin-like molecules," Proceedings of the National Academy of Sciences of the United States of America, vol. 98, no. 2, pp. 502-506, 2001.

[12] J. C. Engert, M.-C. Vohl, S. M. Williams et al., " 5 ' Flanking variants of resistin are associated with obesity," Diabetes, vol. 51, no. 5, pp. 1629-1634, 2002.

[13] S. D. Patel, M. W. Rajala, L. Rossetti, P. E. Scherer, and L. Shapiro, "Disulfide-dependent multimeric assembly of resistin family hormones," Science, vol. 304, no. 5674, pp. 1154-1158, 2004.

[14] I. N. Holcomb, R. C. Kabakoff, B. Chan et al., "FIZZ1, a novel cysteine-rich secreted protein associated with pulmonary inflammation, defines a new gene family," The EMBO Journal, vol. 19, no. 15, pp. 4046-4055, 2000.

[15] P. Codoñer-Franch and E. Alonso-Iglesias, "Resistin: insulin resistance to malignancy," Clinica Chimica Acta, vol. 438, pp. 46-54, 2015.

[16] W. An, Y. Bai, S.-X. Deng et al., "Adiponectin levels in patients with colorectal cancer and adenoma: a meta-analysis," European Journal of Cancer Prevention, vol. 21, no. 2, pp. 126-133, 2012.

[17] S. P. Gialamas, T. N. Sergentanis, C. N. Antonopoulos, N. Dessypris, G. P. Chrousos, and E. T. Petridou, "Circulating leptin levels and risk of colorectal cancer and adenoma: a casecontrol study and meta-analysis," Cancer Causes and Control, vol. 24, no. 12, pp. 2129-2141, 2013.

[18] R. K. Joshi, W. J. Kim, and S.-A. Lee, "Association between obesity-related adipokines and colorectal cancer: a case-control study and meta-analysis," World Journal of Gastroenterology, vol. 20, no. 24, pp. 7941-7949, 2014.

[19] R. K. Joshi and S.-A. Lee, "Obesity related adipokines and colorectal cancer: a review and meta-analysis," Asian Pacific Journal of Cancer Prevention, vol. 15, no. 1, pp. 397-405, 2014.

[20] X. T. Xu, Q. Xu, J. L. Tong et al., "Meta-analysis: circulating adiponectin levels and risk of colorectal cancer and adenoma," Journal of Digestive Diseases, vol. 12, no. 4, pp. 234-244, 2011.

[21] F. Tulubas, R. Mete, M. Oznur, and B. Topcu, "The role of adipocytokines in colon cancer and adenomas," Journal of Medical Biochemistry, vol. 33, no. 2, pp. 135-142, 2014.

[22] A. Sălăgeanu, C. Tucureanu, L. Lerescu et al., "Serum levels of adipokines resistin and leptin in patients with colon cancer," Journal of Medicine and Life, vol. 3, no. 4, pp. 416-420, 2010.

[23] T. E. Nakajima, Y. Yamada, T. Hamano et al., "Adipocytokines as new promising markers of colorectal tumors: adiponectin for colorectal adenoma, and resistin and visfatin for colorectal cancer," Cancer Science, vol. 101, no. 5, pp. 1286-1291, 2010.

[24] A. Kumor, P. Daniel, M. Pietruczuk, and E. Małecka-Panas, "Serum leptin, adiponectin, and resistin concentration in colorectal adenoma and carcinoma (CC) patients," International Journal of Colorectal Disease, vol. 24, no. 3, pp. 275-281, 2009.

[25] F. Kosova, T. Coskun, Y. Kaya, E. Kara, and Z. Ari, "Adipocytokine levels of colon cancer patients before and after treatment," Bratislava Medical Journal, vol. 114, no. 7, pp. 394-397, 2013.

[26] G. Gonullu, H. Kahraman, A. Bedir, A. Bektas, and I. Yücel, "Association between adiponectin, resistin, insulin resistance, and colorectal tumors," International Journal of Colorectal Disease, vol. 25, no. 2, pp. 205-212, 2010.
[27] E. Danese, M. Montagnana, A. M. Minicozzi et al., "The role of resistin in colorectal cancer," Clinica Chimica Acta, vol. 413, no. 7-8, pp. 760-764, 2012.

[28] L. Lu, L. Yi, M. Qiling, and H. Peizhen, "Study on serum leptin, adiponectin and resistin concentration in patients with colorectal cancer," Journal of Medical Research, vol. 39, no. 9, pp. 78-80, 2010.

[29] D. Wägsäter, M. Mumtaz, S. Lofgren, A. Hugander, and J. Dimberg, "Resistin in human colorectal cancer: increased expression independently of resistin promoter $-420 \mathrm{C}>\mathrm{G}$ genotype," Cancer Investigation, vol. 26, no. 10, pp. 1008-1014, 2008.

[30] S. Otake, H. Takeda, S. Fujishima et al., "Decreased levels of plasma adiponectin associated with increased risk of colorectal cancer," World Journal of Gastroenterology, vol. 16, no. 10, pp. 1252-1257, 2010.

[31] G. Y. F. Ho, T. Wang, M. J. Gunter et al., "Adipokines linking obesity with colorectal cancer risk in postmenopausal women," Cancer Research, vol. 72, no. 12, pp. 3029-3037, 2012.

[32] A. Hillenbrand, J. Fassler, N. Huber et al., "Changed adipocytokine concentrations in colorectal tumor patients and morbidly obese patients compared to healthy controls," BMC Cancer, vol. 12, article 545, 2012.

[33] D. F. Stroup, J. A. Berlin, S. C. Morton et al., "Meta-analysis of observational studies in epidemiology: a proposal for reporting. Meta-analysis of Observational Studies in Epidemiology (MOOSE) group," JAMA, vol. 283, no. 15, pp. 2008-2012, 2000.

[34] G. A. Wells, B. Shea, D. O'Connell et al., "The Newcastle-Ottawa Scale (NOS) for assessing the quality of nonrandomised studies in meta-analyses," http://www.ohri.ca/programs/clinical _epidemiology/oxford.asp.

[35] S. P. Hozo, B. Djulbegovic, and I. Hozo, "Estimating the mean and variance from the median, range, and the size of a sample," BMC Medical Research Methodology, vol. 5, article 13, 2005.

[36] T. Liu, G. Li, L. Li, and P. Korantzopoulos, "Association between C-reactive protein and recurrence of atrial fibrillation after successful electrical cardioversion: a meta-analysis," Journal of the American College of Cardiology, vol. 49, no. 15, pp. 1642$1648,2007$.

[37] A. Tarkowski, J. Bjersing, A. Shestakov, and M. I. Bokarewa, "Resistin competes with lipopolysaccharide for binding to tolllike receptor 4," Journal of Cellular and Molecular Medicine, vol. 14, no. 6, pp. 1419-1431, 2010.

[38] L. R. Howe, K. Subbaramaiah, C. A. Hudis, and A. J. Dannenberg, "Molecular pathways: adipose inflammation as a mediator of obesity-associated cancer," Clinical Cancer Research, vol. 19, no. 22, pp. 6074-6083, 2013.

[39] H. Mu, R. Ohashi, S. Yan et al., "Adipokine resistin promotes in vitro angiogenesis of human endothelial cells," Cardiovascular Research, vol. 70, no. 1, pp. 146-157, 2006.

[40] N. Di Simone, F. Di Nicuolo, M. Sanguinetti et al., "Resistin regulates human choriocarcinoma cell invasive behaviour and endothelial cell angiogenic processes," The Journal of Endocrinology, vol. 189, no. 3, pp. 691-699, 2006.

[41] M. Qatanani, N. R. Szwergold, D. R. Greaves, R. S. Ahima, and M. A. Lazar, "Macrophage-derived human resistin exacerbates adipose tissue inflammation and insulin resistance in mice," The Journal of Clinical Investigation, vol. 119, no. 3, pp. 531-539, 2009.

[42] T. Mahmoudi, K. Karimi, M. Arkani et al., "Resistin $-420 \mathrm{C}>\mathrm{G}$ promoter variant and colorectal cancer risk," The International Journal of Biological Markers, vol. 29, no. 3, pp. e233-e238, 2014. 
[43] A. Fernández-Sánchez, E. Madrigal-Santillán, M. Bautista et al., "Inflammation, oxidative stress, and obesity", International Journal of Molecular Sciences, vol. 12, no. 5, pp. 3117-3132, 2011.

[44] E. Orgel and S. D. Mittelman, "The links between insulin resistance, diabetes, and cancer," Current Diabetes Reports, vol. 13, no. 2, pp. 213-222, 2013.

[45] A. G. Renehan, M. Zwahlen, C. Minder, S. T. O’Dwyer, S. M. Shalet, and M. Egger, "Insulin-like growth factor (IGF)-I, IGF binding protein-3, and cancer risk: systematic review and metaregression analysis," The Lancet, vol. 363, no. 9418, pp. 13461353, 2004.

[46] Y. Ikeda, H. Tsuchiya, S. Hama, K. Kajimoto, and K. Kogure, "Resistin affects lipid metabolism during adipocyte maturation of 3T3-L1 cells,” FEBS Journal, vol. 280, no. 22, pp. 5884-5895, 2013.

[47] O. A. Mojiminiyi and N. A. Abdella, "Associations of resistin with inflammation and insulin resistance in patients with type 2 diabetes mellitus," Scandinavian Journal of Clinical and Laboratory Investigation, vol. 67, no. 2, pp. 215-225, 2007.

[48] M. Filková, M. Haluzík, S. Gay, and L. Šenolt, “The role of resistin as a regulator of inflammation: implications for various human pathologies," Clinical Immunology, vol. 133, no. 2, pp. 157-170, 2009.

[49] S. Riondino, M. Roselli, R. Palmirotta, D. Della-Morte, P. Ferroni, and F. Guadagni, "Obesity and colorectal cancer: role of adipokines in tumor initiation and progression," World Journal of Gastroenterology, vol. 20, no. 18, pp. 5177-5190, 2014. 


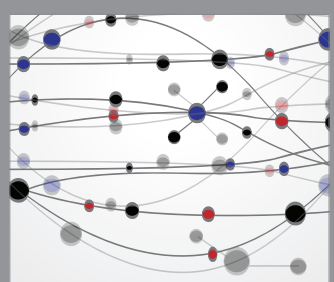

The Scientific World Journal
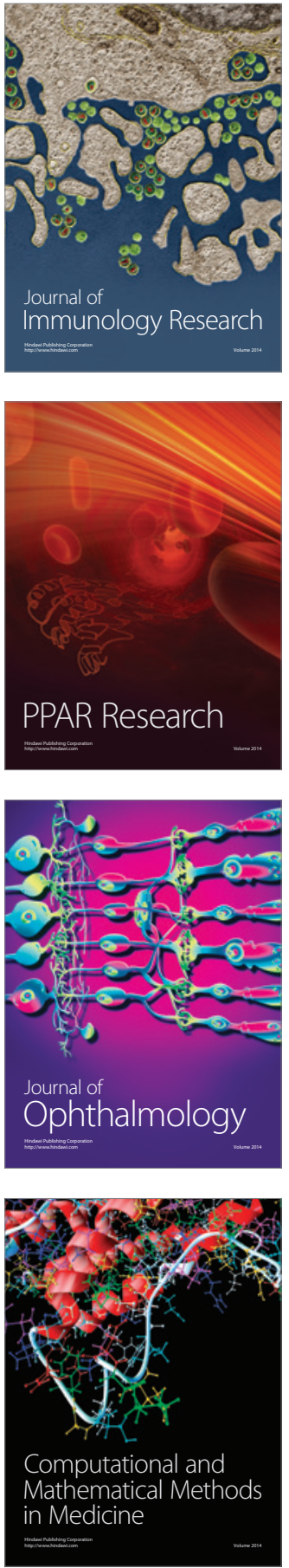

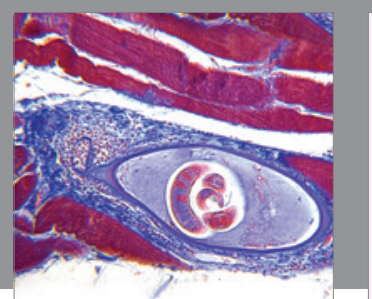

Gastroenterology Research and Practice

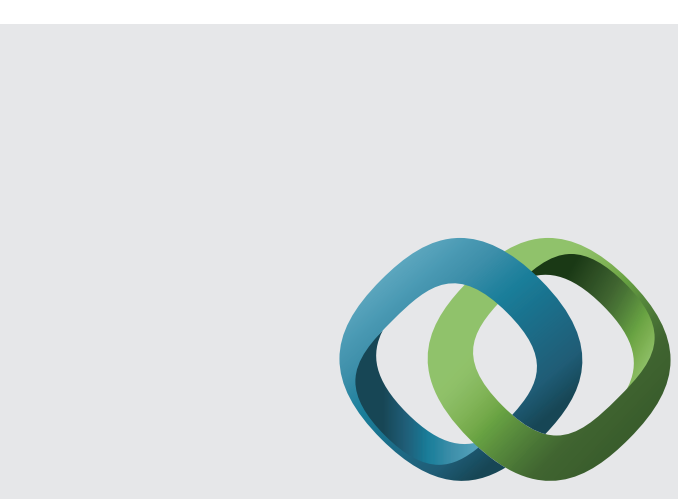

\section{Hindawi}

Submit your manuscripts at

http://www.hindawi.com

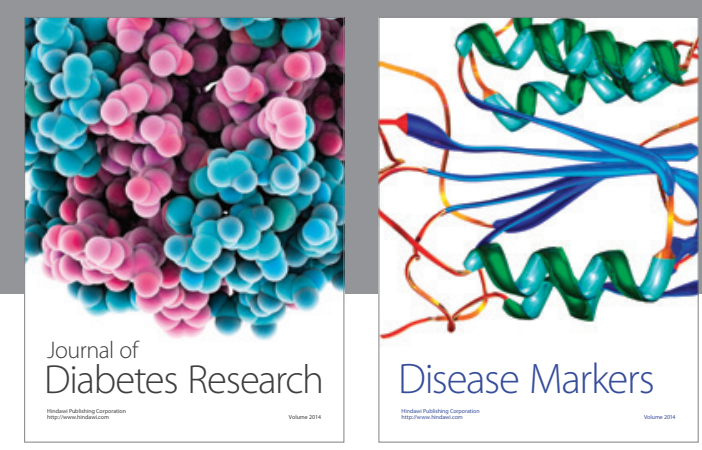

Disease Markers
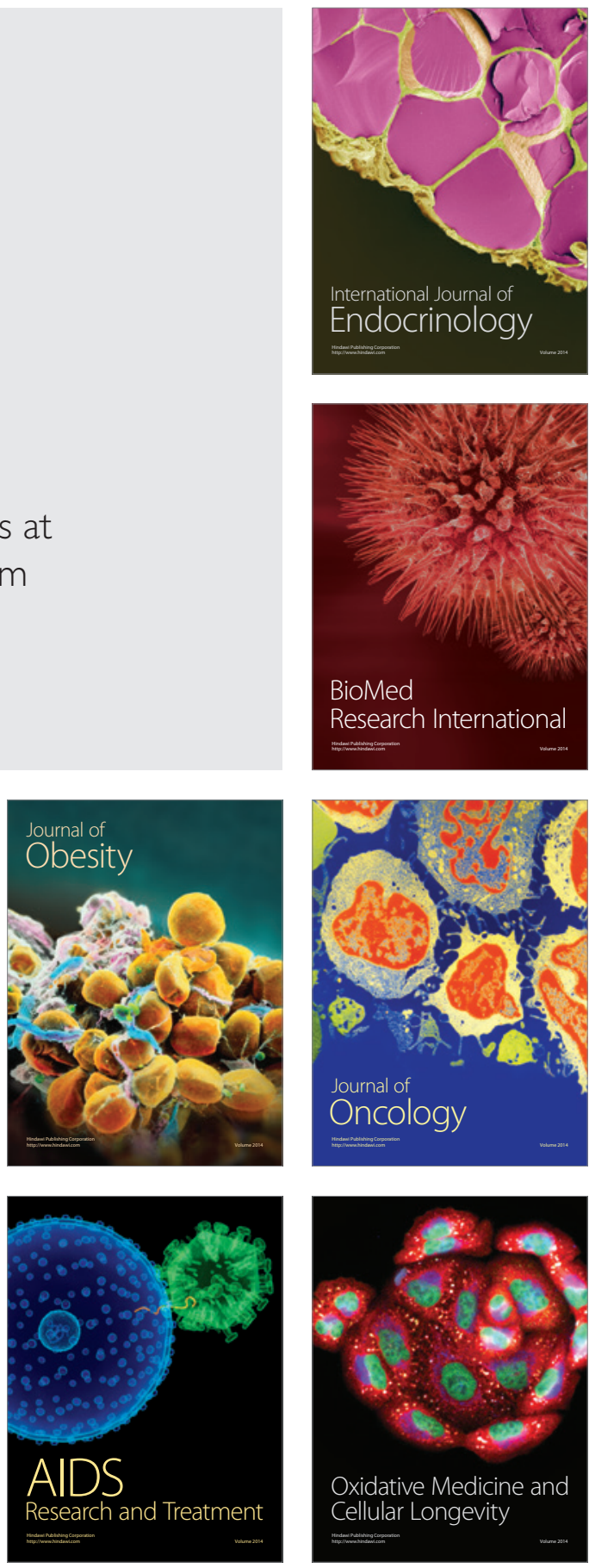